\title{
Bilioenteric Fistula - Not any more a contraindication for Laparoscopic Cholecystectomy
}

\author{
Samiron Kumar Mondal ${ }^{1}$, Sharmistha Roy ${ }^{2}$
}

\begin{abstract}
Background: Bilioenteric fistulas include cholecystoduodenal fistula, cholecystocolic fistula, and cholecystogastric fistula. They are known but rare complication of cholecystitis. Previously presence of any cholecystoenteric fistula was an indication to convert laparoscopic cholecystectomy to open operation ${ }^{1}$. But with time and experience new techniques, new technology and expertise has developed in the laparoscopic field and now the scenario is changed. This is our experience in a tertiary care diabetic hospital where we encountered 21 cases of cholecystoenteric fistula during laparoscopic cholecystectomy over a period of 2 years. Our series: From January 2013 to January 2015 we performed 1191 cases of laparoscopic cholecystectomy in our unit. We encountered 12 cases of cholecystoduodenal fistula, 7 cases of cholecystocolic fistula and 2 cases of cholecystogastric fistula. All cases were diagnosed during laparoscopy, and were dealt with laparoscopy. Intracorporeal suturing was used in all cases.
\end{abstract}

Observation: Successful per operative diagnosis requires suspicion and careful adhesiolysis in all cases. Avoiding conversion to open operation reduces hospital stay, pain, wound infection and chest complications associated with upper abdominal incision. As the fistulous tract is usually near the fundus and far away from Callots there is not much increased risk of common bile duct injury.

Conclusion: Cholecystoenteric fistulas can now safely be managed laparoscopically in advanced centers by experienced surgeon.

Key Words: Bilioenteric/cholecystoenteric, Intracorporeal, callots

\section{Introduction}

Bilioenteric/cholecystoenteric fistula is a condition where there is an abnormal fistulous tract between the gallbladder and gastrointestinal tract. The communication most frequently occurs between the fundus of the gallbladder and duodenum, but other parts of the gallbladder like body or infundibulum may be involved. Other parts of the gut like right side of transverse colon and stomach may also take part in fistula formation less frequently. The incidence of such complication ranges from $3-5 \%$ in patients with cholelithiasis according to some articles ${ }^{2}$, while other reports suggest an incidence of $0.15 \%-4.8 \%$ of patients undergoing biliary surgery $^{3-6}$. However it is a rare complication of chronic cholecystitis and is associated with gallstone in at least $75 \%$ of cases ${ }^{7}$. It may also be associated with Mirrizi syndrome ${ }^{8}$.

Since 1987 laparoscopic cholecystectomy (LC) has been the favored treatment for gallbladder diseases ${ }^{9}$. With experience gained from laparoscopic surgery, LC has successfully been

1. Dr. Samiron Kumar Mondal, FCPS (Surgery), Associate Professor, BIRDEM General Hospital \& IMC, Dhaka 1000, Bangladesh.

2. Dr. Sharmistha Roy, FCPS (Surgery), Assistant Professor, BIRDEM General Hospital \& IMC, Dhaka 1000, Bangladesh.

\section{Corresponding Author:}

Dr Samiron Kumar Mondal

Associate Professor of Surgery

BIRDEM General Hospital \& Ibrahim Medical College

Dhaka 1000, Bangladesh

E-mail: drshamiron@yahoo.com attempted in every kind of gallbladder disease. Previous reports suggest ${ }^{1,10}$ cholecystoenteric fistula as an indication to convert laparoscopy to open operation, but with increased experience in laparoscopic field, development of technology like stapling device and techniques like intra corporeal suturing and knotting, cholecystoenteric fistula is no more a contra indication of laparoscopic cholecystectomy.

Our series: Working in a tertiary care diabetic hospital has given us the opportunity to deal with a large volume of patients, specially complicated ones referred from diabetic centers all over the country. In our unit 1191 laparoscopic cholecystectomy was done between January 2013 and January 2015. We found 21 cases of cholecystoenteric fistulas. In our series the incidence was about $1.7 \%$. Out of them, 12 cases of cholecystoduodenal fistula, 7 cases of cholecystocolic fistula and 2 cases of cholecystogastric fistula. All cases were diagnosed during laparoscopy, and were dealt with laparoscopy.

Patient Profile: The age range of patients were between 43-69 years. 3 female and 18 male patient. Symptoms were similar to that of chronic cholecystitis, but in all cases duration of history were more than 3 years, suggesting repeated attack and inflammation precipitating fistula formation. Pre operative USG showed contracted Gallbladder in 9 cases. None of them suggested Pneumobilia.

Operative details: Dense pericholecystic adhesion was found in all cases. After working out through omental adhesion, gallbladder was found to be densely adherent to duodenum or colon or stomach. Careful blunt dissection 
delineated the fistulous tract eventually, which was then transfixed, dismantled from the gallbladder side and undermined into the gut by continuous suture. A 2/0 round vicryl was used in all cases. A drain was kept in Morrison's pouch in all cases. Significant fibrosis were also present in the Callot's in 13 cases. Gall bladder was delivered in endo bag in all cases. Average operating time was 60-80 minutes.

Post operative management: A nasogastric drain was kept in patients with cholecysto duodenal and cholecysto gastric fistula for at least 3 days. Oral feeding then gradually started, liquid from $4^{\text {th }}$ POD and normal from $5^{\text {th }}$ day. Drain was removed after oral feeding started. Patient was discharged with oral medication on $5^{\text {th }}$ or $6^{\text {th }}$ POD.

\section{Discussion}

Since advent of laparoscopic surgery untiring efforts, innovation and invention of enthusiastic technology loving laparoscopic surgeons has obviated most of the initial contraindications of laparoscopic cholecystectomy. In last 15 years several articles have been published regarding complicated cases of laparoscopic cholecystectomy and their safety issue. Most of the recent reports are very encouraging, indicating improved results with experience. Cholecystoenteric fistula has long been a reason to convert laparoscopic cholecystectomy to an open one, but recent reports from different parts of the world shows the condition can safely be managed by by laparoscopy. In our series we had 21 cases of cholecystoenteric fistula, all diagnosed peroperatively. In some reports preoperative diagnosis has been made in up to $50 \%$ of cases $^{7}$. Presence of pneumobilia on USG, CT Scan, passage of contrast to colon on ERCP, delineation of fistula tract by MRCP T2 image has been reported ${ }^{11,12}$. But chlecystoenteric fistula cases mostly have similar clinical feature like chronic cholecystitis $^{13}$, so on what basis such costly investigations are advised or whether it will be cost effective when the incidence rate is $<5 \%$, is not clearly mentioned.

Regarding operative technique most surgeons used stapling device to disengage fistula tract, which is easier, faster, avoids peritoneal soiling ${ }^{14-18}$. In our series we have used hand sewn technique, simply because we work in a developing country with limited resource. Intracorporeal suturing is a bit time consuming but it's cheaper and end results are equally good ${ }^{19}$. We have employed warm saline wash to the peritoneal cavity to wash out biliary soiling and kept a drain as required.

Post operative results are very rewarding, showing significant decrease in post operative pain, and chest complications associated with open surgery. Less pain causes early mobilization and less post operative paralytic ileus. Oral feeding from as early as $2^{\text {nd }}$ POD has been reported in cases where stapling device is used ${ }^{8}$.

Results of long term follow up is not available in our series as we followed up our early cases up to 3 months, but in studies with long term (2.4 years) follow up ${ }^{8}$ results are good.

\section{Conclusion}

Diagnosis of Cholecystoenteric fistula pre operatively and per operatively requires a high index of suspicion and experience.
In the hands of a surgeon with significant laparoscopic experience, laparoscopic cholecystectomy and laparoscopic management of cholecystoenteric fistula is safe and effective.

\section{References}

1. Malik A, Laghari AA, Talpur KAH, Memon A, Mallah Q, Memon JM. Laparoscopic cholecystectomy in empyema of gall bladder: An experience at Liaquat University Hospital, Jamshoro, Pakistan. J Minim Access Surg. 2007 Apr-Jun; 3(2): 52-6.

2. Angrisani L, Corcione F, Tartaglia F, et al. Cholecystoenteric fistula is not a contraindication for laparoscopic surgery. Surg Endosc 2001;15:1038-41.

3. Haff RC, Wise L, Ballinger WF. Biliary enteric fistulas. Surg Gynecol Obstet 1971; 133: 84-8.

4. Ozdemer A, Atli MY, Coscun T, Ozenk A, Hensek E. Biliary enteric fistulas. Int Surg 1997; 82: 280-3.

5. Zuemer FC, Coffin-Kwart VE, Conway MJ. Biliary enteric fistulas: management of 47 cases in native Americans. Am J Surg 1979; 138: $301-4$.

6. Glenn F, Reed C, Grafe WR. Biliary enteric fistula. Surg Gynecol Obstet 1981;153:527.

7. Wang WK, Yeh CN, Jan YY. Successful laparoscopic management for cholecystoenteric fistula.World J Gastroenterol 2006 February 7; 12(5): 772-5.

8. Chowbey PK, Bandyopadhyay SK et al. Laparoscopic Management of Cholecystoenteric Fistulas; Journal of Laparoendoscopic \& Advanced Surgical Techniques. 2006; 16(5): 467-72.

9. Cuschieri A, Dubois F, Mouiel J, Mouret P, Becker H, Buess G, Trede M, Troidl H. The European experience with laparoscopic cholecystectomy. Am J Surg. 1991; 161: 385-7.

10. Macintyre IM, Wilson RG. Laparoscopic cholecystectomy. Br J Surg. 1993; 80: 552-9.

11. Yamashita H, Chijiiwa K, Ogawa Y, Kuroki S, Tanaka M. The internal biliary fistula--reappraisal of incidence, type, diagnosis and management of 33 consecutive cases. HPB Surg. 1997; 10: 143-7.

12. Yasuhiro H, Takayuki M, Miyoshi F, Yuji M, Hiroyuki K. Laparoscopic treatment of cholecystocolonic fistula: report of a case preoperatively diagnosed by barium enema. Surg Laparosc Endosc Perc Tech 1999; 9: 217-9.

13. Knol JA, Eckhauser FE: Biliary fistulas. In: Zuidema GD, Yeo CJ, Turcotte JG (eds): Shackelford's Surgery of the Alimentary Tract, vol. III, 5th ed. Philadelphia: W.B. Saunders, 2002, pp 273-9.

14. Angrisani L, Corcione F, Tartaglia A, Tricarico A, Rendano F, Vincenti R, Lorenzo M, Aiello A, Bardi U, Bruni D, et al. Cholecystoenteric fistula (CF) is not a contraindication for laparoscopic surgery. Surg Endosc 2001; 15: 1038-41.

15. Sharma A, Sullivan M, English H, Foley R. Laparoscopic repair of cholecystoduodenal fistulae. Surg Laparosc Endosc 1994; 4: 433-5.

16. Yeh CN, Jan YY, Liu NJ, Yeh TS, Chen MF. Endo-GIA for ligation of dilated cystic duct during laparoscopic cholecystectomy: an alternative, novel, and easy method. J Laparoendosc Adv Surg Tech A 2004; 14: 153-7.

17. Martin I, Siriwardena A. Safe laparoscopic cholecystectomy in the presence of a cholecysto-enteric fistula. Dig Surg 2000; 17: 178-80.

18. Prasad A, Foley RJ. Laparoscopic management of cholecystocolic fistula. Br J Surg. 1994; 81: 1789-90.

19. Prasad A, Kapur R. Cholecystogastric fistula: laparoscopic repair. Surg Laparosc Endosc Perc Tech 2001; 11: 277-8. 